\title{
ENHANCEMENT OF BILE DUCT ULTRASOUND IMAGES FOR CHOLANGIOCARCINOMA SURVEILLANCE
}

\author{
Pichet Wayalun $^{1}$, Saiyan Saiyod ${ }^{2 *}$, Nittaya Chamadol ${ }^{3}$ \\ ${ }^{1,2}$ Department of Computer Science, Faculty of Science \\ ${ }^{3}$ Department of Rediology, Faculty of Medicine, \\ ${ }^{3}$ Liver Fluke and Cholangiocarcinoma Research Center \\ Khon Kaen University, Khon Kaen 40002, Thailand \\ Email: pichet.w@kkumail.com¹, saiyan@kku.ac.th², nittayachamadol@yahoo.com³ \\ DOI: https://doi.org/10.22452/mjcs.vol31 no4.1
}

\begin{abstract}
Bile Ducts Cancer (Cholangiocarcinoma: CCA) is a serious public health problem. The surgical resection is one of the curative treatments for CCA. The surgical resection in the early stage of the CCA gives a good chance for recovery; therefore, early detection in the surgical resection stage is of a critical importance. Periductal fibrosis $(P D F)$ in the bile duct is one factor which is considered about CCA in Thailand. It can be detected using the liver ultrasound images; however, the liver ultrasound images contain speckle noise which decreases PDF detection performance. This paper proposes a new method for enhancement of bile duct ultrasound image (EBDU). The main idea of the EBDU method is to reduce the speckle noise and enhance clear structures of the liver ultrasound images. The proposed method consists of three processes which are the speckle reduction, edge preservation, and intraregion smoothing. The speckle reduction is performed in frequency domain to filter out the high frequency and low frequency noise out of the ultrasound image by using the bandpass filter. The criterion of the bandpass filter is the size of the structures in the ultrasound image, which could be large structures (mirrored edges) and small structures (smoothing). Edge preservation is then performed by applying the statistics filter to distinctly increase the boundary structure; it sorts out all pixel values from the surrounding neighborhood into a numerical order. Finally, intraregions of the liver ultrasound images are smoothed by intra-region smoothing process which can diffuse the homogeneous region. The enhanced liver ultrasound images were tested by computer-aided detection on PDF regions $(C A D P D F)$. Variety of liver ultrasound images were used. To evaluate and compare the performance of the proposed method, four measurements are applied which are the scan line pixel (SLP), the Slope-Smooth Speckle Index (SLSI), Equivalent number of looks (ENL), Speckle suppression index (SSI), and the accuracy achieved through the PDF regions segmentation. The experimental results show that the proposed method is able to give higher success rate of enhancing liver ultrasound images compared to other methods. Moreover, the accuracy of CADPDF system was evaluated. The proposed EBDU method and other methods are applied in CADPDF system. The experimental results show that the proposed EBDU method achieved better performance than other methods, giving an accuracy of $80.36 \%$.
\end{abstract}

Keywords: Speckle reduction; Periductal Fibrosis; Image Processing; Ultrasound imaging; Bile duct.

\subsection{INTRODUCTION}

Cholangiocarcinoma (CCA) is a bile duct cancer which is a major public health problem in Thailand and its neighboring countries. The risk factor of the CCA in Thailand is liver fluke, which may cause bile duct inflammation and subsequently leads to the Periductal fibrosis (PDF). The thickness and echoic wall that runs in parallel to the portal vein [1] are the characteristic of the PDF. The liver fluke has high prevalence in northeast Thailand, and it has infected more than one-third of a population of 20 million [2]. Currently, PDF in the liver ultrasound images are used to identify risk groups of CCA [1]. 
The liver ultrasound images are attempted to be utilized for surveillance of the CCA because of their low cost, no side effects, easy mobility, and real time formation. Nowadays, approximately 16,000 to 20,000 affected individuals per year go to take ultrasound for the CCA surveillance that tends to increase [1]. Therefore, the computer system is developed as a tool for the CCA surveillance by using the liver ultrasound images data in order to analyse the PDF. It is called "computer-aided detection the PDF regions" (CADPDF) [3]. However, liver ultrasound images are of a poor quality because they contain the speckle noise which is bigger than the spatial resolution of liver ultrasound images. The speckle noise affects the important regions, texture and boundaries. As a result, the quality of liver ultrasound images are degenerated [4] [5]. Therefore, the liver ultrasound images must be enhanced by speckle reduction. De-speckle noise is defined as the speckle noise reduction.

The ultrasound image enhancement methods have been developed using filters which were based on time and frequency domains. The filters that have been developed based on the time domain are Shao [6], Wong [7], Zhang [8], Coup'e [9], and $\mathrm{Yu}$ [10]. The mentioned filters have been developed by applying the bilateral filtering, Monte Calo filter, Fuzzy subpixel, NL-means filter, Blockwise technique, respectively. These filters can reduce the added noise but not good enough to filter the multiplicative noise which is the noise model of the ultrasound images. The ultrasound filters have been developed based on the frequency domain to reduce the multiplicative noise in the ultrasound images. Elamvazuthi filter [11] and Tsantis filter [7] have been developed based on the frequency domain utilizes the Daubechies, Wiener, Anisotropic diffusion, back-propagation tracking and singularity detection of wavelet transform modulus maxima. They can reduce the noise but not preserve sharp features and the important regions in the ultrasound images.

In addition, the enhancement method is one part of the diagnosis system for ultrasound images. The important features such as edge, shape and brightness should be improved. Yang [12] developed the technique of the shape symmetry between the malignant and benign breast tumors that applied the Gaussian filters to enhance the ultrasound images. However, Yang's technique can only effectively reduce additive noise. Bodzioch [13] developed the automated contours extraction technique on the gallbladder ultrasound images, applying the Rank filters for reduction of speckle noise. Gomez [14] developed the automated segmentation technique on the breast ultrasound images which applied the morphological and the Anisotropic method to improve the shape of the features. Joo [15] proposed the computer-aided diagnosis (CAD) technique for identifying breast nodule malignancy. The technique utilizes the Median filtering, Unsharp masking, contrast enhancement to ultimately enhance the features of the images. Many researches have shown the way to enhance the ultrasound images, this include developing the filter based on the frequency domain, preserving the edge features by using non-linear operator and improving the important regions by using the intensity diffusion method.

This paper proposes a hybrid technique for the enhancement of liver ultrasound images for the PDF region through reduction of speckle noise. The proposed method applies the FFT filter to reduce the speckle noise in the frequency domain by the bandpass filter, which cuts the speckle noise frequencies by using the low and high pass filter. The method demonstrated best performance in reducing the speckle noise, but it did not preserve the edges of the image. Thus, the median filter is applied to reduce the aggravating noise in the PDF region boundary, a region which contained significant amount of noise. In addition, the rough nature of the PDF region is one of the problems for the PDF region detection accuracy. The roughness is an effect of the ultrasound wave reflection on the moving organ. Hence, anisotropic diffusion is used to smoothen the features of the PDF region. As a result, the proposed technique showed the best performance to enhance the liver ultrasound image for the PDF region, which is a new contribution in the field. Moreover, our method increases the accuracy of PDF regions detection system. The organization of the paper is as follows: the problems of the liver ultrasound images and the performance measurement are addressed in Section 2; Section 3 introduces the proposed method; and in Section 4, the proposed method is compared with other enhancement methods; lastly, some conclusions are given with a summary in Section 5.

\subsection{MATERIAL AND METHOD}

The liver ultrasound images are poor quality, and contain speckle noise, blurred edges, and none smooth "rough" regions. As a result, the detection performance degrades. Therefore, the ultrasound images should be enhanced. In this section, the problems will be defined, including the liver ultrasound images quality and measurements technique. After that, the filter methods will be presented. 

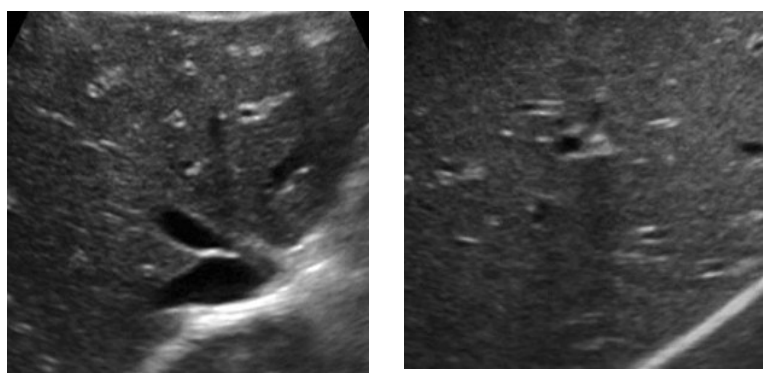

Fig. 1: Liver ultrasound images

\subsection{Problem definition}

\subsubsection{Liver ultrasound image}

The liver ultrasound images are used in the CCA surveillance (Fig.1). They are obtained from the ultrasound device, which reflects the coherent ultrasound wave on the liver. The ultrasound waves reflect on a non-smooth organs'

surfaces. Movement of the ultrasound device affects the image in the same manner, producing rough images. As a result, the liver ultrasound images comprise speckle noise which is the problem for the liver ultrasound images. The speckle noise is the major image quality degradation factor that contains multiplicative noise function and additive noise function. The liver ultrasound images and noise model are shown in equation (1) [1]:

$$
f(x, y)=g(x, y) \cdot \eta_{m}(x, y)+\eta_{n}(x, y)
$$

where $f(x, y)$ is the noise within the image, $g(x, y)$ is the original image presented without noise, $\eta_{m}(x, y)$ is a multiplicative noise function, and $\eta_{n}(x, y)$ is an additive noise function. Commonly, the effect of the multiplicative noise is more significant than the effect of the additive noise [16-18]. As mentioned previously, the speckle noise is problem of the liver ultrasound image; it is the characteristic of the multiplicative noise. Therefore, in this paper, the additive noise is ignored. The effect of the multiplicative noise on the liver ultrasound images include speckle, edge blur, and non-smooth regions within the liver ultrasound image. PDF images' size and color, as well as the boundaries of the PDF region which are blurred by the multiplicative noise also affect the detection performance, leading to its degradation.

\subsubsection{Measurements}

The performance measure that has been used to evaluate the ultrasound image enhancement method is the despeckle values. The sample measurement techniques of the enhancement quality are the signal-to-noise ratio (SNR) $[6][7,9]$, the Mean preservation and Variance reduction [10], the ultrasound de-speckling assessment index $(\tilde{Q})$
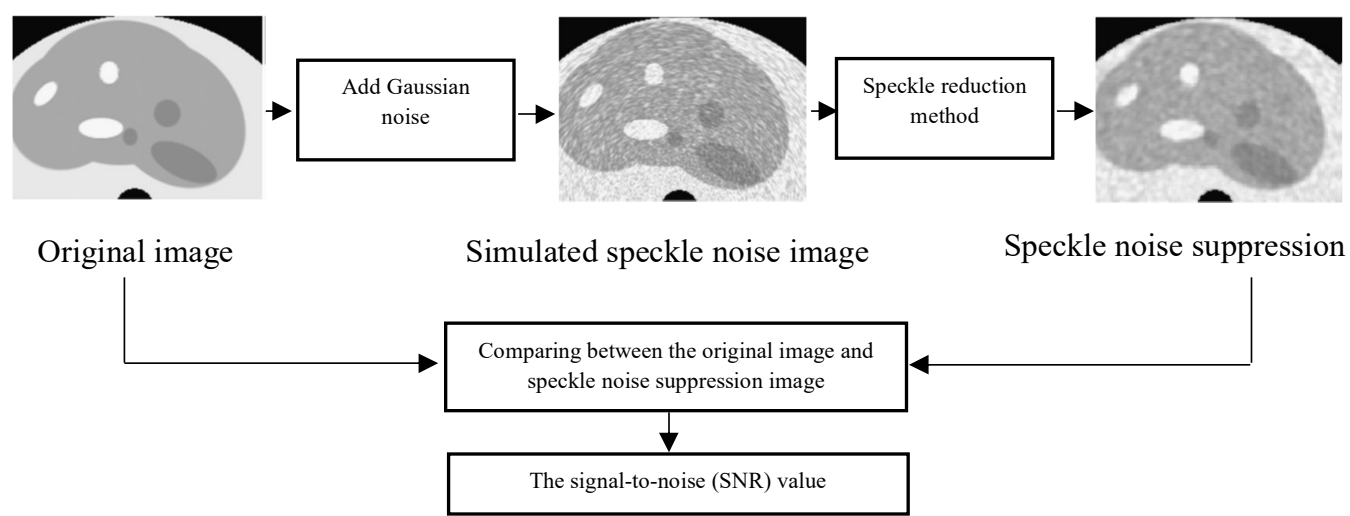

Fig. 2: Estimating performance for speckle noise reduction by SNR [17] 
[9], the Speckle index (SI). These are the performance measurements of the smooth speckle noise. The quality of the edge structure of ultrasound images is measured by the Pratt's Figure of merit (PFOM) $[6,10][8], \eta_{\text {[7] }}$ and $\beta$ [16]. There are several measurement techniques for original image comparison with the filtered image (Fig.2). However, an ultrasound image already contains the speckle noise; hence, the measurement techniques that are mention above are not suitable for measuring the enhancement techniques of ultrasound images.

\subsection{Transformation and filter methods}

\subsubsection{The fast Fourier transform (FFT)}

Signal analysis is important for the image processing. A fast Fourier transform (FFT) is one of the tools used for signal analysis. FFT is a fast algorithm of DFT; however, the inverse discrete Fourier transform (IDFT) is an inversion of DFT. DFT and IDFT can convert the time domain to the frequency domain and vice versa [19] [20].

\subsubsection{FFT filter}

FFT filter is one of the filter methods used for image processing; it filters out the noise between the large structures and small structures in frequency domain. It is utilized as a plugin for ImageJ; it includes three methods which are Fourier transform, Difference of Gaussian (DoG), and inverse Fourier transform [21].

\subsubsection{The median filter method}

Median filter (MDF) is one of classical filter methods that is applied in the image processing and other fields. It is a nonlinear method, and is based on the order statistics theory. The MDF is used in many fields such as image processing and communication. It can smooth out the signal and reduce noise in the images which contain speckle noise, salt and paper noise, and impulsive noise. MDF substitutes the pixel's value that is being considered, by the middle pixel's value [11] [17] [22] [23].

\subsubsection{The anisotropic diffusion method}

Anisotropic diffusion is a nonlinear method for contrast enhancement and noise reduction. The concept of the anisotropic diffusion method is to compute the diffusion for the homogeneous regions, and finding the energy function which has the minimum for energy-dissipating process. The anisotropic diffusion can effectively reduce additive noise and speckle in images. In addition, the anisotropic diffusion preserves the edges of the image [10,24] [25]. Filter methods have been presented to improve the image performance for improving PDF detection; however, these methods will be modified, as illustrated in next section.

\subsection{PROPOSED METHOD}

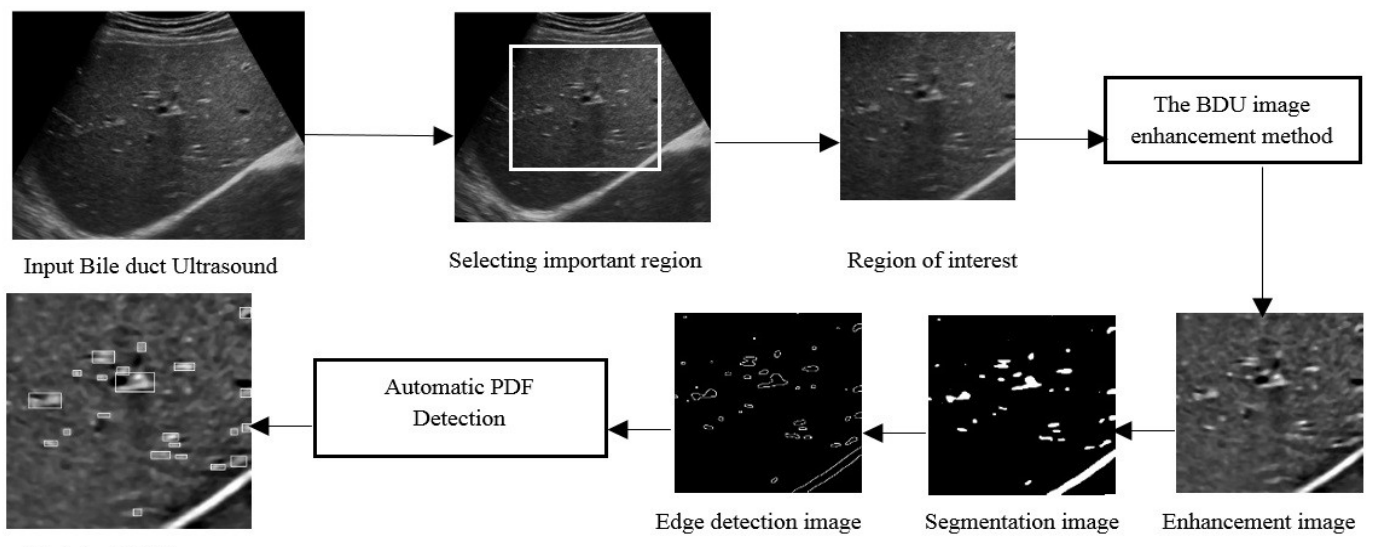

Detected PDF image

Fig. 3: Proposed CADPDF system 


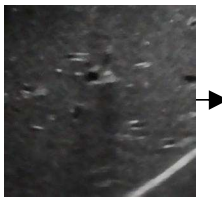

Input image
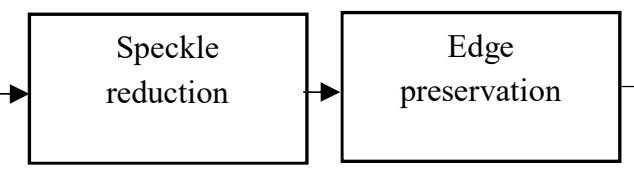

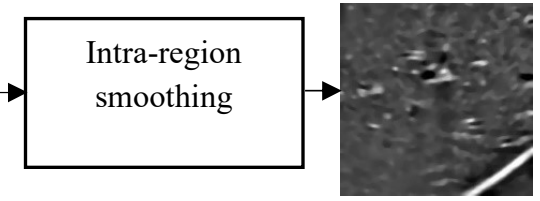

Output image

Fig. 4: Flowchart of the proposed method for enhancement the liver ultrasound image

The Computer-aided detection of the PDF (CADPDF) is a system for identifying the PDF regions for CCA surveillance [3]. The CADPDF system is shown in Fig. 3; it consists of three steps: firstly, the liver ultrasound image is enhanced by noise filter; secondly, the regions of foreground image are segmented; thirdly, the region edges are detected; finally, automatic PDF detection detects the PDF regions in the liver ultrasound images. However, if the original liver ultrasound images are ran in CADPDF system, the detection accuracy is not good, because the liver ultrasound images are poor in quality; therefore, the liver ultrasound images must be enhanced. In this paper, a proposed new technique to enhance the liver ultrasound images that is called "the enhancement of bile duct ultrasound images" (EBDU). The EBDU is developed based on the speckle noise reduction, edge preservation, and intra-regions smoothing. The EBDU flowchart is shown in Fig. 4.

\subsection{Speckle reduction}

The speckle noise is a serious problem in the liver ultrasound images; it occurs from the ultrasound wave reflection. It scatters and affects the important regions of the liver ultrasound image. Therefore, the liver ultrasound image quality degrades. For this reason, this paper has developed the speckle reduction method based on the frequency domain. The $f_{i}[x, y]$ is a pixel of the liver ultrasound image of $[x, y]$ coordinate that contains speckle noise. $f_{i}[x, y]$ transforms time domain to frequency domain, which is given as equation (2) [20]:

$$
F[p, q]=\frac{1}{\sqrt{M N}} \sum_{x=0}^{M-1} \sum_{y=0}^{N-1} f_{i}[x, y] \cos \left(\frac{2 \pi p x}{M}\right)-f_{i}[x, y] j \sin \left(\frac{2 \pi q y}{N}\right), p=0,1, \ldots, M-1 ; q=0,1, \ldots, N-1
$$

where $M$ and $N$ are the size of the liver ultrasound image. The liver ultrasound image on the frequency domain is $F \square$, which called Fourier image. The process of liver ultrasound image transformation from time domain to frequency domain is shown in Fig.5. Then, $F[]$ is multiplied by the transfer function of a DoG method [21] which is the bandpass filter. These are shown in equation (3) [21]:

$$
F[p, q]=F[p, q] .\left(\exp \left[-\left(\frac{2 S_{\text {small }}}{L} k\right)^{2}\right]\left\{1-\exp \left[-\left(\frac{2 S_{\text {large }}}{L} k\right)^{2}\right]\right\}\right)
$$

where $L$ is edge length, $S_{\text {small }}$ and $S_{\text {large }}$ are the lower bound and the upper bound of bandpass filter. In this paper, $S_{\text {small }}$ and $S_{\text {large }}$ are 3 and 40 pixel, respectively. $k$ is the distance of the current pixel of the centre of the Fourier image, calculated by $k=\sqrt{k_{x}^{2}+k_{y}^{2}}$. Equation 3 is performed in "Filter out large and small structure by bandpass filter" as shown in Fig. 5. Finally, the Fourier image is transformed from frequency domain to time domain as shown in equation (4) [20]:

$$
f_{s}[x, y]=\frac{1}{\sqrt{M N}} \sum_{x=0}^{M-1} \sum_{y=0}^{N-1} F[p, q] \cos \left(\frac{2 \pi p x}{M}\right)+F[p, q] j \sin \left(\frac{2 \pi q y}{N}\right), p=0,1, \ldots, M-1 ; q=0,1, \ldots, N-1
$$

Using this process, the liver ultrasound image is converted to time domain as well as original image. The inversion process of liver ultrasound image is shown in Fig.5. Though the liver ultrasound image is enhanced by speckle 
reduction method, the region edges of the liver ultrasound image are not clear enough; thus, the region's edges of the liver ultrasound image must be enhanced.

\subsection{Edge preservation}

The region's edges of the liver ultrasound image are the important data for the CADPDF system, albeit unclear. These edges are blurred because of the multiplicative noise that causes degradation to the edge detection performance; thus, this paper improves the edge preservation method to enhance the region edges. The edge preservation method is performed based on the order statistics theory. It sorts all pixel values of the surrounding neighborhood into the numerical order. And then, the pixel values are replaced by the pixel being considered with the middle pixel value. The edge preservation is shown in equation (5) [11]:

$$
f_{e}[x, y]=\operatorname{median}_{i, j \in N_{x y}}\left\{f_{s}[x, y]\right\}
$$

where $f_{e}[x, y]$ and $f_{s}[x, y]$ are the filter pixel and noise pixel of the neighborhood area $N_{x y} \cdot i, j$ represent the window size of filter that are 3 and 3, respectively. The equation 5 is performed in "Preserving strong edges using the edge preservation" as shown in Fig.5. Although the region of the liver ultrasound image is enhanced, the intraregions of the region are not smooth enough, which is one of the problems to degenerate the performance of the CADPDF system.

\subsection{Intra-regions smoothing}

The intra-region quality of the region for liver ultrasound images is one of the factors that affect accuracy of the segmentation of the region from background liver ultrasound images, but the intra-regions are not smooth enough, foreground looks similar to background, this degrades the region segmentation performance. Therefore, the intraregions must be enhance. This paper proposes intra-regions smoothing technique which can enhance the intra-region of the region for liver ultrasound images. This technique diffuses the homogeneous region of the region for the liver ultrasound image. The intra-region smoothing method is shown in equations (6-9) [10].

$$
\frac{\partial f_{e}[x, y]}{\partial t}=\operatorname{div}\left(c\left(\mid \nabla f_{e}[x, y]\right) \nabla f_{e}[x, y]\right)
$$

where $\nabla$ is the gradient operator with respect to the space variables, $d i v$ is divergence operator, $c\left(\mid \nabla f_{e}[x, y]\right)$ is diffusion coefficient, and $f_{e}[x, y]$ is output images. Moreover, the inter-regions smoothing can be rewritten in the form of nonlinear, partial differential equations on continuous domain as:

$$
\left\{\begin{array}{c}
\frac{\partial f_{e}[x, y]}{\partial t}=\operatorname{div}\left(c\left(\mid \nabla f_{e}[x, y]\right) \nabla f_{e}[x, y]\right) \\
f_{e}[x, y](t=0)=f_{e}[x, y]
\end{array}\right.
$$

and the diffusion coefficients function is presented by [10] [24] as:

$$
c\left(\mid \nabla f_{e}[x, y]\right)=\frac{1}{1+\left(\frac{\left|\nabla f_{e}[x, y]\right|}{k}\right)^{2}}
$$

where $k$ is the edge magnitude parameter. The region edges are detected by gradient magnitude which is $\left|\nabla f_{e}[x, y]\right|$. The diffusion conditions for the intra-region smoothing are as follows: the small intensity gradient can 
block the diffusion across the region edges as "If $\left|\nabla f_{e}[x, y]\right| \geq k$, then $c\left(\mid \nabla f_{e}[x, y]\right) \rightarrow 0$ "; the diffusion can cross the liver ultrasound image edges as "If $\left|\nabla f_{e}[x, y]\right| \leq k$, then $c\left(\mid \nabla f_{e}[x, y]\right) \rightarrow 1$ ".

In addition, the intra-regions smoothing can be rewritten in the discrete form which is applied in this paper as:

$$
f_{o}[x, y]=f_{e}[x, y]+\frac{\sum_{p \in \eta_{s}} c\left(\nabla f_{e}[x, y]\right) \nabla f_{e}[x, y]}{\left|\eta_{s}\right|}
$$

where $f_{o}[x, y]$ is the output image from the intra-region smoothing, and $f_{e}[x, y]$ is the liver ultrasound image. The spatial neighborhood of pixel is $\eta_{s}$, and the numbers of pixel in the windows are $\left|\eta_{s}\right|$ which is four except at the image boundaries. $\nabla f_{e}[x, y]$ is the gradient of the liver ultrasound image that is computed as: $\nabla f_{e}[x, y]=$ $f_{e}[x, y]-f_{p}[x, y], p \in \eta_{s} \cdot c\left(\nabla f_{s, p}[x, y]\right)$ is diffusion coefficient. $f_{p}[x, y]$ is boundaries image.

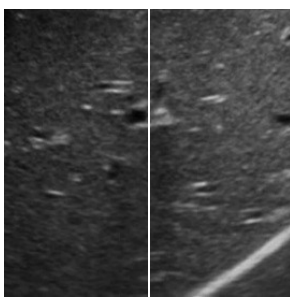

(a) Vertical

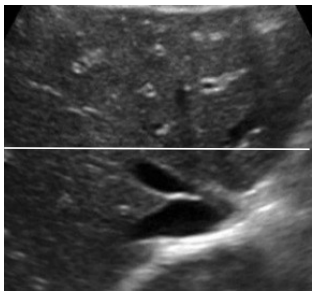

(b) Horizontal

Fig. 6: The scan line pixel technique

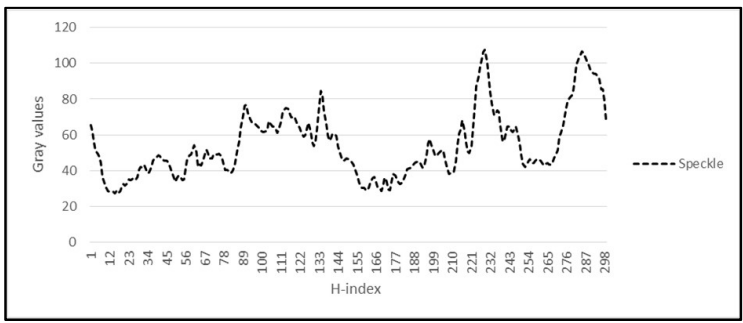

Fig. 7: The gray values of H-index from Fig.6 (a)

\subsection{ENHANCMENT PERFORMANCE EVALUATION}

The performance of the proposed EBDU method is evaluated between the original liver ultrasound image and the enhanced liver ultrasound image. The EBDU is compared to Shao [6], Coupé [26], and $\mathrm{Yu}$ [10] in four methods utilizing real liver ultrasound images. In addition, the Slope-Smooth Speckle Index (SLSI), Equivalent number of looks (ENL), Speckle suppression index (SSI), and accuracy of segmentation the PDF region by the CADPDF system [27] are the efficiency metric of all enhancement methods.

\subsection{The scan line pixel technique (SLP)}

To clearly evaluate each method for the liver ultrasound image enhancement, this paper uses the scan line pixel technique (SLP) $[28,29]$ to evaluate the de-speckle noise. SLP is considered by scan line running through the liver ultrasound image. The gray values can be obtained along the scan line index of the liver ultrasound image. The scan 
line index represents the pixel coordinate on the scan line that consist of $\mathrm{V}$-index and $\mathrm{H}$-index, which are the pixel coordinate along the vertical line (Fig. 6a) and horizontal line (Fig.6b) of liver ultrasound image, respectively. This technique can represent the performance of each method such as de-speckle performance, the edge preservation, and brightness of the liver ultrasound image. Fig. 6 shows a sample of the scan line pixels, and Fig.7 shows the gray value of scan line pixel technique.

\subsection{Slope-smooth speckle index (SLSI)}

The slope-smooth speckle index (SLSI) can measure the de-speckle of the liver ultrasound image. If the slope value is high, it means that the de-speckle is low in performance. In contrast, if the slope value is low, it means that the despeckle is high in performance. So that, the SLSI is utilized to evaluate the de-speckle performance of the liver ultrasound images. The SLSI consist of two process: firstly, the slope values of SLP on the enhancement liver ultrasound image is calculated by equation (10) [30]; finally, cumulative sum method [31] is applied to present the de-speckle performance. The SLSI is shown in equation (10-11) [31]: 
Input: liver ultrasound image $f_{i}[x, y], M=300, N=300$; dimension of the liver image, $L$; edge length of structures , $S_{\text {small }}=3, S_{l \arg e}=40, I$ and $j=3$; window size of edge preservation, $N_{x y}$; the neighbourhood area, $k$; edge magnitude parameter

Output: Enhancement of liver image $f_{o}[x, y]$

\section{Begin}

for $P=0 \rightarrow M-1$ do

for $q=0 \rightarrow N-1$ do

for $x=0 \rightarrow M-1$ do

for $y=0 \rightarrow N-1$ do

$F[p, q] \leftarrow f_{i}[x, y] \cos \left(\frac{2 \pi p x}{M}\right)-f_{i}[x, y] i \sin \left(\frac{2 \pi q y}{N}\right)$

Convert the liver ultrasound image form time domain to frequency domain

End

End

End

End

for $P=0 \rightarrow M-1$ do

for $q=0 \rightarrow N-1$ do

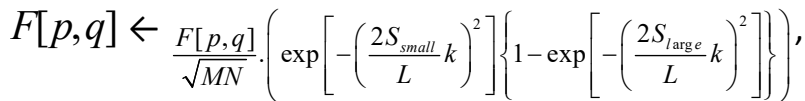

Filter out the large and small structures by bandpass filter

End

End

for $x=0 \rightarrow M-1$ do

for $y=0 \rightarrow N-1$ do

for $P=0 \rightarrow M-1$ do

for $q=0 \rightarrow N-1$ do

$f_{s}[x, y] \leftarrow F[p, q] \cos \left(\frac{2 \pi p x}{M}\right)+F[p, q] i \sin \left(\frac{2 \pi q y}{N}\right)$,

Invert the liver ultrasound images from End

End

End

End

for $x=0 \rightarrow M-1$ do

for $y=0 \rightarrow N-1$ do

$$
f_{e}[x, y] \leftarrow \underset{i, j \in N_{x y}}{\operatorname{median}}\left\{\frac{f_{s}[x, y]}{\sqrt{M N}}\right\},
$$

Preserve the strong edges

End

End

$\nabla f_{e}[x, y] \leftarrow f_{e}[x, y]-f_{p}[x, y], p \in \eta_{s}$,

Find the liver gradient image

$c\left(\nabla f_{e}[x, y]\right) \leftarrow$

$$
\frac{1}{1+\left(\frac{\left|\nabla f_{e}[x, y]\right|}{k}\right)^{2}}
$$

Find the diffusion coefficients

for $x=0 \rightarrow M-1$ do

for $y=0 \rightarrow N-1$ do

$$
f_{r}[x, y] \leftarrow c\left(\nabla f_{e}[x, y]\right) \nabla f_{e}[x, y]
$$

End

End $f_{o}[x, y] \leftarrow f_{e}[x, y]+\frac{f_{r}[x, y]}{\left|\eta_{s}\right|}, \quad \begin{aligned} & \text { Make the diffusion of the homogeneous } \\ & \text { regions }\end{aligned}$

End

Proposes EBDU Algorithm using the speckle noise reduction, edge preservation, and intra-region smoothing

Fig. 5: Proposed EBDU algorithm. 


$$
M_{i}=\left|\frac{y_{i+1}-y_{i}}{(i+1)-i}\right|=\left|y_{i+1}-y_{i}\right|
$$

where $M_{i}$ is the slope value of $i$ index. $y$ is the gray value of $i$ index.

$$
\operatorname{SLSI}(i)=\sum_{i=0}^{I} M_{i}
$$

where $S L S I(i)$ is the cumulative sum of slope values of $i$ index . I size of image.

\subsection{Accuracy of the PDF regions' segmentation}

The enhancement of liver ultrasound images is one of the processes of the CADPDF system. If the appropriate enhancement method of liver ultrasound image is applied, the accuracy of the PDF regions segmentation will be achieved [29] [32] [33] [34]. So that, the PDF regions segmentation is used to evaluate the performance of the enhancement of liver ultrasound images. The results of segmentation will be counted and confirmed by an expert. Accuracy is expressed as shown in equation (12) [3]:

$$
\text { Accuracy }=\frac{A}{B} X 100
$$

where $A$ and $B$ are correct segments of the PDF regions and overalls segments of the liver ultrasound image, respectively as shown in Fig.8.

\subsection{Equivalent number of looks}

Equivalent number of looks (ENL) [35] is the measurement of the de-speckle performance of images. It can describe the de-speckle of the image. ENL is defined as the ratio between the mean squared to the variance as shown in equation (13) [35]:

$$
E N L_{\text {value }}=\left(\frac{\mu_{f}}{\rho_{f}}\right)^{2}
$$

where $\mu_{f}$ and $\rho_{f}$ are the mean squared values and variance values of the image, respectively. The higher value of $E N L_{\text {value }}$, the better de-speckle performance.

\subsection{Speckle suppression index}

Speckle suppression index (SSI) [36] is one of the measurements of the performance of the de-speckle noise. It is defined by the coefficient ratio of variance filter, which normalizes the variance of the enhanced image and original image as follows equation (14) [36]:

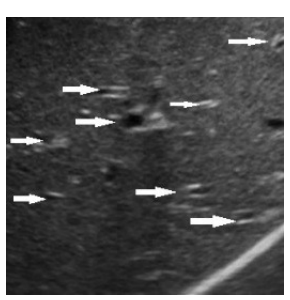

(a) The PDF regions in the liver ultrasound image

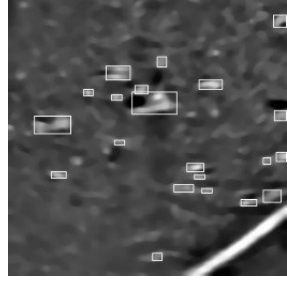

(b) Overall segmentation of the PDF regions

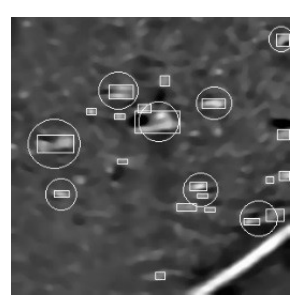

(c) Correct segmentation of the PDF regions

Fig. 8: Accuracy of the PDF regions segmentation 


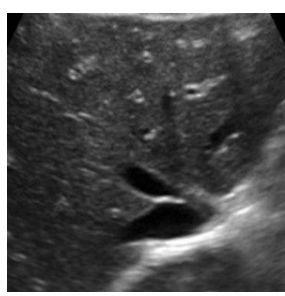

(a) Original

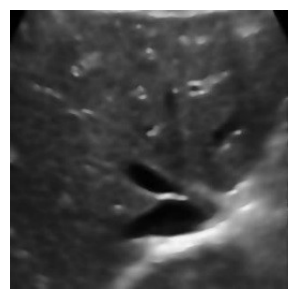

(b) $\mathrm{Yu}$

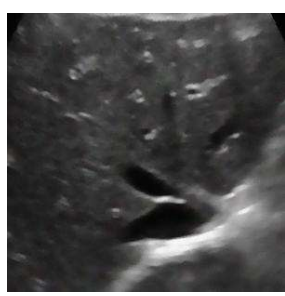

(c) Shao

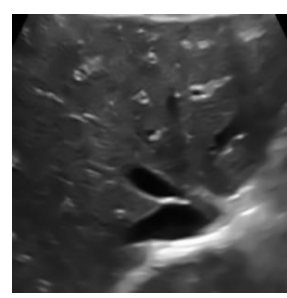

(d) Coupé

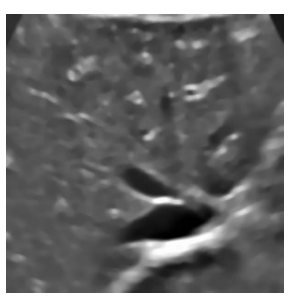

(e) Proposed EBDU

Fig. 9: Results of each enhancement method on the PE001 image

$$
S I_{\text {value }}=\frac{\sqrt{\rho_{f}}}{\mu_{f}} \cdot \frac{\sqrt{\rho_{o}}}{\mu_{o}}
$$

where $\rho_{f}$ and $\rho_{o}$ are the variance of enhanced image and original image, $\mu_{f}$ and $\mu_{o}$ are the mean of enhanced image and original image. The smaller the $S_{S I_{v a l u e}}$ is, the better performance of de-speckle noise.

\subsection{EXPERIMENTAL RESULTS}

In this section, the performance evaluations of the liver ultrasound enhancement method EBDU are compared to Shao [6], Coupé [26], and Yu [10] using SLP, SLSI, ENL, SSI, and accuracy of the PDF regions segmentation. The proposed method has been developed using Matlab and visual $\mathrm{C}++$ program. The hardware specifications involved in our experiment were a PC with $3.40 \mathrm{GHz}$, CPU Intel(R), and 8 GB RAM.

\section{$5.1 \quad$ Test image dataset}

The test ultrasound images used in our experiment were accurately explained by an expert radiologist. The 30 ultrasound images are liver ultrasound images of 30 different patient. In addition, each liver ultrasound image has PDF regions, which are scattered in the image. Each liver ultrasound image size is $300 \times 300$ pixel. Furthermore, the liver ultrasound images are supported by the project "Application of multiple biomarkers for screening of opisthorchiasis-related hepatobiliary diseases in the high risk area for Cholangiocarcinoma in Khon Kaen province".

\subsection{De-speckle noise performance}

The de-speckle noise performance of the proposed EBDU method, Shao, Coupé, and Yu methods are compared by using the same liver ultrasound image, which is called PE001 image and PE002 image. PE001 image and PE002 image are one liver ultrasound image of the datasets. The parameters are defined as follows. Shao method defined

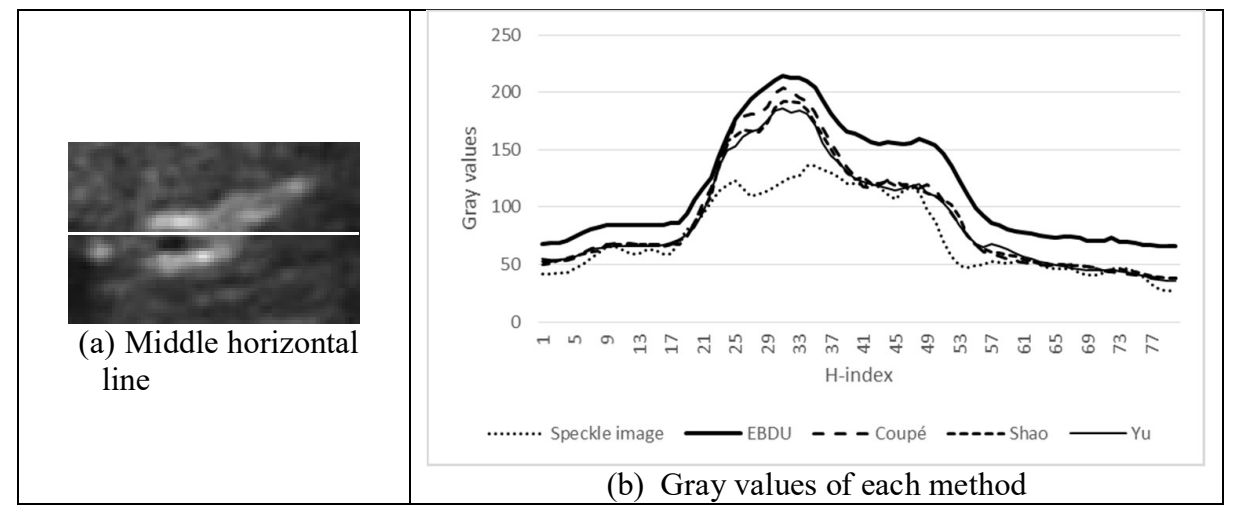

Fig. 10: The de-speckle performance of each enhancement method by using the scan line pixel technique 


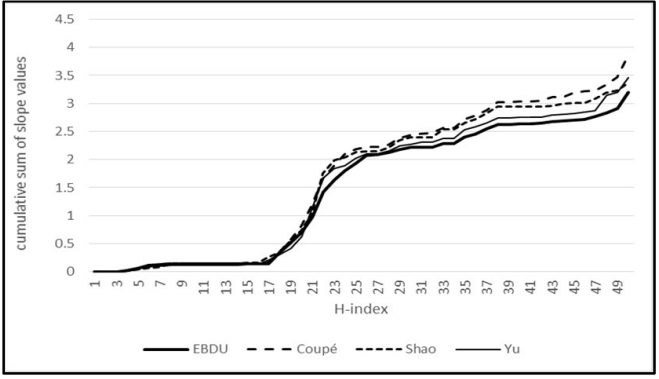

(a) SLSI method

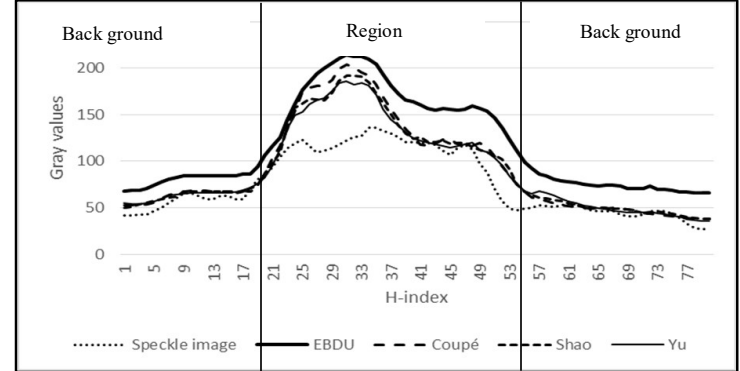

(b) SLP method

Fig. 11: Performance comparison for each method

the mask size as $5 \times 5$ pixels, $\sigma_{1}=5$, and $\sigma_{2}=0.1$. Coupé method defined the mask size as $7 \times 7$ pixels, and $\alpha=3$. Yu method defined the mask size as $7 \times 7$ pixels. The proposed EBDU method defines the $S_{\text {small }}=3, S_{\text {large }}=40$ and the mask size as $3 \times 3$ pixels. In addition, the iterations number and smoothing per iterations number are 20 and 1 , respectively.

\subsubsection{The visual results of the de-speckle performance of the various methods}

The enhancement performance of each method on the PE001 images are shown in Fig. 9. The Yu method output can preserve the image texture and edges, but it does not sufficiently reduce speckle noises as shown in Fig. 9(b).

$\mathrm{Yu}$ method is better in de-speckling than Shao method. Moreover, the important regions and edges of the liver ultrasound image appear better than Yu method. Coupé method provides good de-speckling performance, but the intra-regions of the liver ultrasound image are not smooth enough and are low in contrast. EBDU method provides better de-speckling than other mentioned methods. In addition, intra-regions of the liver ultrasound images are smooth and high in contrast.

\subsubsection{Results of de-speckle performance by SLP and SLSI}

SLP considers the grayscale values to measure the de-speckle performance. The gray values can be obtained from middle horizontal line of the PE001 image as shown in Fig. 10(a). Then, gray values of each method are plotted as shown in Fig. 10(b). SLP can demonstrate the de-speckle performance, edge preservation, and brightness. The despeckle performance is considered by line smoothing. If the considered line is smooth, it means that the de-speckle is high in performance. In addition, the slope of line can represent the edge preservation performance. The high slope means that edge is preserved. The brightness is illustrated by the range of gray level values of background and regions. The high range means good brightness. In addition, SLSI is used to measure the performance of de-speckle for the liver ultrasound images. If the slope value is low, it means that the de-speckle is high in performance. 
Table 1: Values of the de-speckle performance index SSI and ENL for each method on the PE002 image

\begin{tabular}{|l|l|l|l|l|}
\cline { 2 - 5 } \multicolumn{1}{c|}{} & \multicolumn{2}{c|}{ Vertical } & \multicolumn{2}{c|}{ Horizontal } \\
\hline Methods & SSI & ENL & SSI & ENL \\
\hline Coupé & 0.00613 & 10.26245 & 0.00870 & 2.67435 \\
\hline Shao & 0.00611 & 10.23728 & 0.00886 & 2.51701 \\
\hline Yu & 0.00610 & 10.22787 & 0.00862 & 2.74561 \\
\hline EBDU & $\mathbf{0 . 0 0 3 6 3}$ & $\mathbf{2 8 . 5 5 3 5 6}$ & $\mathbf{0 . 0 0 6 8 1}$ & $\mathbf{4 . 2 1 0 6 4}$ \\
\hline
\end{tabular}

The de-speckle performance of the proposed EBDU method is better than Yu method while Yu method outperforms the Shao method regarding the same parameter. On the other hand, the Coupe method has the least performance compared to other methods. EBDU method smoothness appears to be better than the other methods. The smoothness of each method is shown in Fig. 11(a-b) which is obtained from the SLSI method and SLP method. Moreover, the performance of region smoothness can be measured by SLSI method. The EBDU method presents the best results in terms of regions smoothness compared to other methods as shown in Fig. 12(a).

Edge preservation and brightness are the important factors to increase the quality of the liver ultrasound images. The edge preservation performance of proposed EBDU method is better than other methods because the slope of line of proposed EBDU method is at a higher degree than other methods as shown in Fig. 11(b). In addition, brightness of the proposed EBDU method is better than Coupe method while Coupe outperforms Shao method. The brightness of $\mathrm{Yu}$ method is less than other methods. In terms of the range of gray level for background and region, the proposed EBDU method presents the highest value compared to the other methods as shown in Fig. 12(c-d).

The order of performance comparison for each method can be summarized as EBDU, Coupé, Shao, and Yu, respectively. The EBDU method has good performance for de-speckling, edge preservation, and brightness enhancement, because the EBDU method filters the speckle noise using the bandpass filter on the frequency domain to analyze and reduce the speckle. In addition, the order statistics theory is applied for edge preservation of the regions for liver ultrasound image. It can enhance the regions' edges of the liver ultrasound image. The intra-regions of liver ultrasound images are smoothed by the diffusion for the homogeneous region which can enhance the quality of the liver ultrasound image region, such as region smoothing and brightness.

\subsubsection{Results of de-speckle performance by SSI and ENL}

In order to evaluate de-speckle performance, the four different enhancement methods are applied on the liver ultrasound image, which is the PE002 image. The SSI and ENL can measure the de-speckle performance. The gray value can be obtained from the middle horizontal line and the vertical line of the PE002 image as show in Fig.13. The gray values of horizontal line or vertical line will be used to compute the $S S I_{\text {value }}$ and $E N L_{\text {value }}$ by equation (13) and equation (14), respectively. Low value of the $S S I_{\text {value }}$ means that the de-speckle is high in performance. The evaluation metric of each method are computed as shown in Table 1. Table 1 shows the performance evaluation of each method on the same liver ultrasound image, which is PE002 image. The order of de-speckle performance can

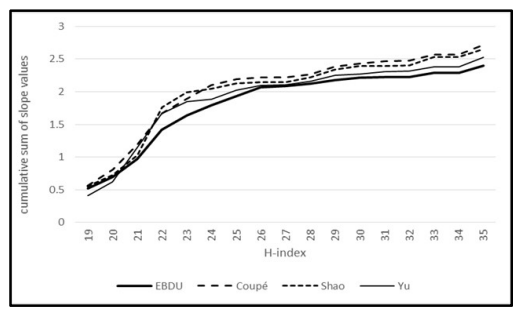

(a) Region

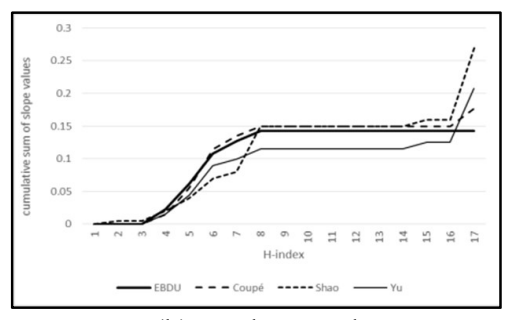

(b) Background

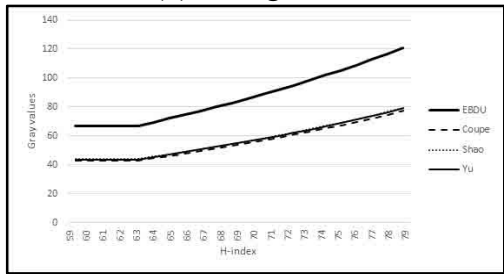

(d) Background gray values of index 59-79

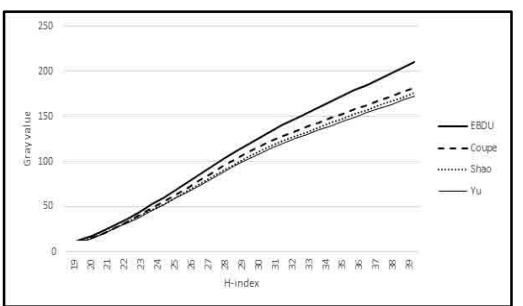

(c) Region gray values of index 19-39

Fig. 12: De-speckle and brightness performance comparison of each method for SLSI method Malaysian Journal of Computer Science. Vol. 31(4), 2018 
be concluded as EBDU, Coupé, Shao, and Yu, respectively. The experimental results show that the EBDU method achieved the small $S S I_{\text {value }}$ and the largest $E N L_{\text {value }}$ from the middle horizontal line and vertical line compared to the other methods. It means that the EBDU method can reduce speckle noise on the horizontal line and vertical direction of the PE002 image, better than the other methods. These results indicate that EBDU method can efficiently reduce the speckle noise in both vertical line and horizontal line.

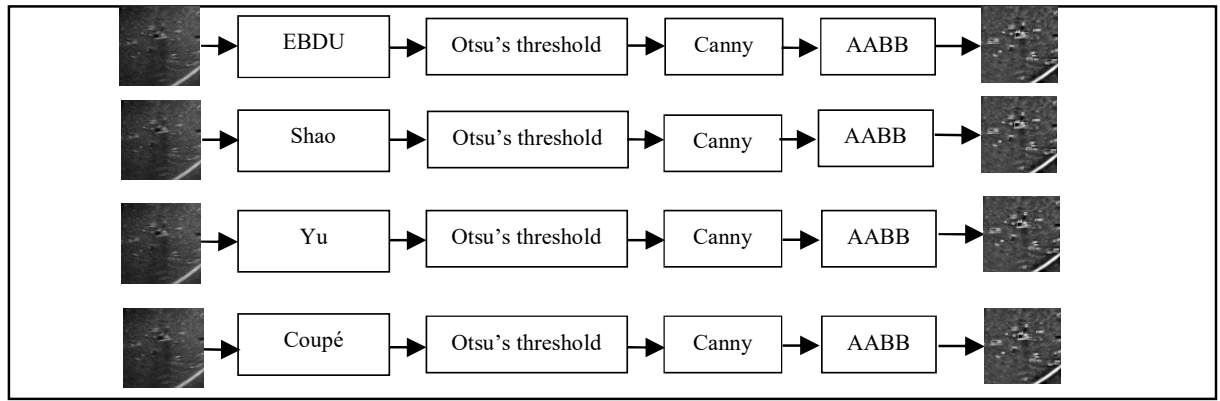

Fig. 14: Proposed CADPDF system performance for each enhancement method

\subsection{The proposed CADPDF system performance}

Table 2: Performance comparison of each enhancement method with the proposed CADPDF system on the different liver ultrasound image

\begin{tabular}{|l|l|}
\hline \multicolumn{1}{|c|}{ Method } & \multicolumn{1}{c|}{ Accuracy (\%) } \\
\hline Coupé & 29.25 \\
\hline Shao & 37.55 \\
\hline Yu & 39.59 \\
\hline EBDU & 80.36 \\
\hline
\end{tabular}

In order to confirm the proposed CADPDF performance, the four different methods are applied to the proposed CADPDF system. In addition, the 30 liver ultrasound images of difference patients are used to compare the performance. The experimental processes can be presented as shown in Fig. 14. Firstly, the liver ultrasound images are enhanced by each method. Secondly, the regions of liver ultrasound image are segmented from background by Otsu method. Thirdly, the region edges of liver ultrasound image are detected by the Canny method [3]. Finally, the PDF regions are identified by the Axis Aligned Bounding Box (AABB) method [3]. The accuracy of each method can be computed by equation (12).

Table 2 indicates the accuracy of PDF region segmentation for the proposed CADPDF system. The experimental

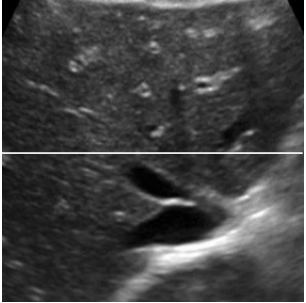

(a) Horizontal line

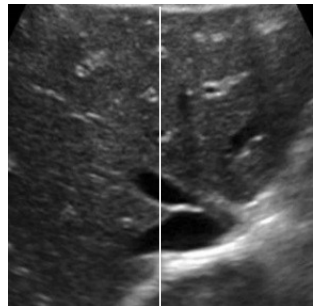

(b) Vertical line

Fig. 13: De-speckle performance measurement by the SSI and ENL on the PE002 image 
results show that the proposed EBDU method can achieve better accuracy than Yu, Shao, and Coup, respectively.

\subsection{CONCLUSION}

The aim of study is to design an enhancement method for the liver ultrasound images involving Cholangiocarcinoma surveillance. This is achieved in the following way: the proposed method for this technique is termed EBDU, which significantly reduces speckle noise without losing structural information of the liver ultrasound images. The proposed EBDU contains speckle reduction, edge preservation, and intra-region smoothing in its process. The speckle reduction process is assessed on the frequency domain. It reduces speckle noise in the liver ultrasound images due to defined lower bound and the upper bound, which are the conditions of the bandpass filter. Edge preservation further enhances the region edges of liver ultrasound image, which preserves the details of the edge. The intra-region smoothing technique smoothens the intra-region of the liver ultrasound images. The proposed EBDU method arguably provided the better results of any individual enhancement methods. Our method reduces the speckle noise, retains the important detail such as the region edges, and improves the brightness of the liver ultrasound image. Therefore, the proposed EBDU method enhances the liver ultrasound images for a better visual quality; hence, it is a good choice for the enhancement of the liver ultrasound images for the CADPDF system.

\section{ACKNOWLEDGMENT}

The Computer and Information Science Interdisciplinary research grant was provided by the Department of romnuter Srionse Khon Kaen Inivarcits 112/2556 Thic studv wras further supported by the Targeted Research Grant, Faculty of Medicine, Khon Kaen University; Grant No. TR54101/PY.

\section{REFERENCES}

[1] N. Chamadol, C. Pairojkul, N. Khuntikeo, V. Laopaiboon, W. Loilome, P. Sithithaworn, and P. Yongvanit, "Histological Confirmation of Periductal Fibrosis From Ultrasound Diagnosis In Cholangiocarcinoma Patients", Journal of Hepato-Biliary-Pancreatic Sciences, Vol. 21, Jan 2014, pp. 316-322.

[2] P. Mairiang, J. Chaiyakum, N. Chamadol., V. Loapaiboon, and M. R. Haswell-elkins, "Cross-Sectional Patterns of Hepatobiliary Abnormalities and Possible Precursor Conditions of Cholangiocarcinoma Associated With Opisthorchis Viverrini Infection In Humans", The American Society of Tropical Medicine and Hygiene, Vol. 55 No. 3, 1996, pp. 298-301.

[3] P. Wayalun, S. Saiyod, and N. Chamadol, "A Hybrid Technique of Noise Reduction with Periductal Fibrosis Ultrasound Images for Periductal Fibrosis Detection System of Cholangiocarcinoma Surveillance", Advanced Materials Research, Vol. 931-932, 2014, pp. 1407-1411.

[4] B. Chen, J.-L. Cai, W.-S. Chen, and Y. Li, "A Multiplicative Noise Removal Approach Based on Partial Differential Equation Model", Mathematical Problems in Engineering, Vol. 2012, pp. 1-15.

[5] G. Mujtaba, L. Shuib, R.G. Raj, R. Rajandram, K. Shaikh, M.A. Al-Garadi, "Automatic ICD-10 multi-class classification of cause of death from plaintext autopsy reports through expert-driven feature selection", PLoS ONE, Vol 12(2): e0170242, 2017. https://doi.org/10.1371/journal.pone.0170242.

[6] D. Shao, P. Liu, and D. C. Liu, "Characteristic Matching-Based Adaptive Fast Bilateral Filter for Ultrasound Speckle Reduction", Pattern Recognition Letters, Vol. 34, 2013, pp. 463-469.

[7] A. Wong and J. Scharcanski, "Monte Carlo despeckling of transrectal ultrasound images of the prostate", Digital Signal Processing, Vol. 22, 2012, pp. 768-775.

[8] Y. Zhang, H.D. Cheng, J. Tian, J. Huang, and X. Tang, "Fractional subpixel diffusion and fuzzy logic approach for ultrasound speckle reduction", Pattern Recognition, Vol. 43, 2010, pp. 2962-2970. 
[9] P. Coup'e, P. Hellier, C. Kervrann, and C. Barillot, "Nonlocal Means-Based Speckle Filtering for Ultrasound Images", IEEE Transactions on Image Processing, Vol. 18 No. 10, 2009, pp. 2221-2229.

[10] Y. Yu and S. T. Acton, "Speckle Reducing Anisotropic Diffusion", IEEE Transactions on Image Processing, Vol. 11 No. 11, 2002, pp. 1260-1270.

[11] I. Elamvazuthi, M. L. B. M. Zain, and K. M. Begam, "Despeckling of ultrasound images of bone fracture using multiple filtering algorithms", Mathematical and Computer Modelling, Vol. 57, 2013, pp. 152-168.

[12] W. Yang, S. Zhang, Y. Chen, W. Li, and Y. Chen, "Shape symmetry analysis of breast tumors on ultrasound images", Computers in Biology and Medicine, Vol. 39, 2008, pp. 231-238.

[13] S. Bodziocha and M. R. Ogielab, "New Approach to Gallbladder Ultrasonic Images Analysis and Lesions Recognition", Computerized Medical Imaging and Graphics, Vol. 33, 2008, pp. pp. 154-170.

[14] W. Gómez, L. Leija, W. C. A. Pereira, and A. F. C. Infantosi, "Semiautomatic contour detection of breast lesions in ultrasonic images with morphological operators and average radial derivative function", Physics procedia, Vol. 3, 2010, pp. 373-380.

[15] S. Joo, Y. S. Yang, W. K. Moon, and H. C. Kim, "Computer-Aided Diagnosis of Solid Breast Nodules: Use of an Artificial Neural Network Based on Multiple Sonographic Features", IEEE Transactions on Medical Imaging, Vol. 23 No. 10, 2004, pp. 1292-1300.

[16] S. Tsantis, N. Dimitropoulos, M. Ioannidou, D. Cavouras, and G. Nikiforidis, "Inter-scale wavelet analysis for speckle reduction in thyroid ultrasound images", Computerized Medical Imaging and Graphics, Vol. 31, 2007, pp. 117-127.

[17] J. L. Mateo and A. Fernández-Caballero, "Finding out general tendencies in speckle noise reduction in ultrasound images", Expert Systems with Applications, Vol. 36, 2009, pp. pp. 7789-7797.

[18] M. I. H. Bhuiyan, M. O. Ahmad, and M. N. S. Swamy, "Spatially adaptive thresholding in wavelet domain for despeckling of ultrasound images", IET Image Processing, Vol. 3 No. 3, 2009, pp. 147-162.

[19] Z. Yuana, F. Li, and M. Hea, "Fast Fourier transform on analysis of Portevin-Le Chatelier effect in Al 5052", Materials Science and Engineering A, No. A530, 2011, pp. 389-395.

[20] N. Ghoshal and J. K. Mandal, "A Novel Technique for Image Authentication in Frequency Domain using Discrete Fourier Transformation Technique (IAFDDFTT)", Malaysian Journal of Computer Science, Vol. 21, No. 1, 2008, pp. 24-32.

[21] G. Chinga, P. O. Johnsen, R. Dougherty, E. L. Berli, and J. Walter, "Quantification of the 3D microstructure of SC surfaces", Journal of Microscopy, Vol. 227, 2007, pp. 254-265.

[22] K. Islam, and R.G. Raj, "Real-Time (Vision-Based) Road Sign Recognition Using an Artificial Neural Network", Sensors, Vol. 17(4), 2017, pp. 853. MDPI AG. http://dx.doi.org/10.3390/s17040853.

[23] R. N. Czerwinska, D. L. Jones, and J. William D. O’Brien, "Ultrasound speckle reduction by directional median filtering", in International Conference on Image Processing 1995, 1995, pp. 358-361.

[24] G. FaDa, T. Phuc, G. ShuaiPing, and Z. LiNa, "Anisotropic diffusion filtering for ultrasound speckle reduction", SCIENCE CHINA Technological Sciences, Vol. 57 No. 3, 2014, pp. 607-614.

[25] M. J. Black, G. Sapiro, D. H. Marimont, and D. Heeger, "Robust Anisotropic Diffusion", IEEE Transactions on Image Processing, Vol. 7 No. 3, 1998, pp. 421-432. 
[26] P. Coup'e, P. Hellier, C. Kervrann, and C. Barillot, "NonLocal Means-based Speckle Filtering for Ultrasound Images", IEEE Transactions on Image Processing, Vol. 19 No. 10, 2009, pp. 2221 - 2229.

[27] A. M. F. Santos, R. M. d. Santos, P. M. A. C. Castro, E. Azevedo, L. Sousa, and J. M. R. S. Tavares, "A novel automatic algorithm for the segmentation of the lumen of the carotid artery in ultrasound B-mode images", Expert Systems with Applications, Vol. 40, 2013, pp. 6570-6579.

[28] T. C. Aysal and K. E. Barner, "Rayleigh-Maximum-Likelihood Filtering for Speckle Reduction of Ultrasound Images", IEEE Transactions on Medical Imaing, Vol. 26 No. 5, 2007, pp. 712-727.

[29] M.-S. Lee, C.-L. Yen, and S.-K. Ueng, "Speckle reduction with edges preservation for ultrasound images: using function spaces approach", IET Image Processing, Vol. 6 No. 7, 2011, pp. 813 - 821.

[30] C. Yang, S. Tian, B. Long, and F. Chen, "Methods of Handling the Tolerance and Test-Point Selection Problem for Analog-Circuit Fault Diagnosis", IEEE Transactions on Instrumentation and Measurement, Vol. 60 No. 1, 2011, pp. 176-185.

[31] M. Cobos, J. J. Perez-Solano, S. Felici-Castell, J. Segura, and J. M. Navarro, "Cumulative-Sum-Based Localization of Sound Events in Low-Cost Wireless Acoustic Sensor Networks", IEEE/ACM Transactions on Audio, Speech, and Language Processing, Vol. 22 No. 12, 2014, pp. 1792-1802.

[32] J. A. Noble and D. Boukerroui, "Ultrasound Image Segmentation: A Survey", IEEE transactions on medical imaging, Vol. 25 No. 8, 2006, pp. 987-1010.

[33] P. Wayalun, S. Saiyod, and N. Chamadol, "A Hybrid Technique of Noise Reduction with Periductal Fibrosis Ultrasound Images for Periductal Fibrosis Detection System of Cholangiocarcinoma Surveillance", in The KKU International Conference 2014 (KKU-IENC 2014), Thailand, 2014, pp. 318-319.

[34] P. Wayalun, S. Saiyod, and N. Chamadol, "A Hybrid Technique for Enhancement of Periductal Fibrosis Ultrasound Images for Cholangiocacinoma Surveillance" in International Conference on Information Technology and Electrical Engineering (ICITEE), 2014, pp. 1-5.

[35] Z. Liu, C. Sun, X. Bai, and F. Zhou, "Infrared Ship Target Image Smoothing Based on Adaptive Mean Shift", in International Conference on Digital lmage Computing: Techniques and Applications (DlCTA), 2014, pp. 1-8.

[36] S. G. Dellepiane and E. Angiati, "Quality Assessment of Despeckled SAR Images", EEE Journal of Selected Topics in Applied Earth Observations and Remote Sensing, Vol. 7 No. 2, 2014, pp. 691-707. 